\title{
Evento adverso no idoso em Unidade de Terapia Intensiva
}

\author{
Adverse event in elderly patients in Intensive Care Unit \\ Evento adverso en personas de edad avanzada en Unidad de Terapia Intensiva
}

\begin{abstract}
Larissa Chaves Pedreira', Adriana Souza Brandão", Aline Macêdo Reis"
' Universidade Federal da Bahia, Escola de Enfermagem, Departamento de Enfermagem Médico-Cirúrgica e Administração em Enfermagem, Campus Universitário do Canela. Salvador-BA, Brasil.

"Universidade Federal da Bahia, Escola de Enfermagem, Curso de Residência em Enfermagem Intensiva Convênio Instituto Sócrates Guanaes e Secretaria de Saúde do Estado da Bahia. Salvador-BA, Brasil.
\end{abstract}

Submissão: 27-02-2011 Aprovação: 01-05-2013

\section{RESUMO}

Objetiva identificar a produção científica, na América Latina, sobre segurança do idoso na UTI e os eventos adversos trazidos pela literatura. Revisão sistemática quantitativa. A coleta, feita no banco de dados do LILACS e SCIELO, considerou descritores indexados e respeitou critérios de inclusão e exclusão. Foram selecionados vinte trabalhos para análise. Em sua maioria, esses trabalhos foram realizados após o ano de 2000, no Brasil, por profissionais enfermeiros e médicos, tratando principalmente de erros com medicação, retiradas acidentais de sondas e cateteres, infecção nosocomial, erro na técnica de procedimento e ocorrência de úlceras por pressão. A produção cientifica sobre a temática ainda é recente na América Latina, sendo preciso atentar para a população idosa nessas unidades, voltando-se para possíveis riscos a que estão expostos.

Descritores: Doença latrogênica; Idoso; Literatura de Revisão como Assunto; Unidade de Terapia Intensiva.

\section{ABTRACT}

This study aims to identify the scientific production in Latin America about the safety of aged people in the ICU and adverse events dealt in the literature. It is a systematic revision, with indexed key words. The inclusion and exclusion criteria were respected. Twenty papers were selected for analysis. Most of them were carried out after the year 2000, in Brazil, by professional nurses and physicians, dealing mostly with adverse events connected to mistakes with medicines, accidental remove of probes and catheters, nosocomial infections, errors in the procedure techniques and occurrence of pressure sores. In the selected papers there were no mentions to adverse events related to behavior changes. The scientific production about the theme is still recent in Latin America, tending to increase. Its necessary to increase the focus to the aged population in these units, taking into account the possible risk to which they are exposed.

Key words: Aged; latrogenic Disease; Intensive Care Units; Review Literature as Topic.

\section{RESUMEN}

El estudio objetiva identificar la producción científica en Latinoamérica sobre la seguridad de las personas mayores en la UTI y los tipos de eventos adversos relatados en la literatura. Trata-se de una revisión sistemática cuantitativa. La colección de datos fue hecha en el banco de datos del LILACS y en la Biblioteca SCIELO, considerándose los países de Latinoamérica a partir de descriptores indexados y respectó criterios de inclusión y exclusión. Fueron seleccionados 20 trabajos para análisis. La mayoría de estos fue realizada después del año 2000, en Brasil, en el Estado de São Paulo, por profesionales enfermeros y médicos, en tratamiento principalmente de eventos adversos conectados a errores con medicación, remoción accidental de sondas y catéteres, infecciones nosocomiales, error en la técnica de procedimiento y de ocurrencia de úlceras de presión. La producción científica sobre la temática és aún reciente en Latinoamérica, tendiendo a aumento. Es necesario ampliar el la mirada para la populación de personas mayores en esas unidades, considerando los posibles riesgos a los que están expuestos.

Palabras clave: Enfermedad latrogénica; Persona Mayor; Literatura de Revisión como Sujeto; Unidad de Terapia Intensiva.

\section{AUTOR CORRESPONDENTE Larissa Chaves Pedreira E-mail: Ichavesp@ufba.br}




\section{INTRODUÇÃO}

É significativo o avanço tecnológico em relação à prevenção, diagnóstico e tratamento de doenças, desenvolvido pela área da saúde nos últimos anos. Os profissionais de saúde se comprometem, desde a sua formação, com a prestação de serviços visando um cuidado de qualidade. Apesar dos esforços na capacitação de pessoal, e na prestação da assistência utilizando toda tecnologia disponível, o paciente pode ser colocado em situações de risco e danos. A partir da década de 70, estudos de revisão em prontuário evidenciaram altas taxas de eventos adversos (EA) relacionados à assistência prestada durante internações hospitalares.

Por EA, define-se lesão não intencional que resultou em incapacidade temporária ou permanente e/ou prolongamento do tempo de permanência ou morte como consequência do cuidado prestado(1).

A segurança do paciente tornou-se preocupação mundial, principalmente após a publicação do livro "Errar é Humano: Construindo um Sistema de Saúde mais Seguro" em 1999(2). Nesse estudo, a estimativa de mortalidade anual, relacionada à EA durante a assistência, foi entre 44.000 e 98.000 pacientes. Na época, uma mortalidade maior que acidentes automobilísticos (43.458), câncer de mama (42.458) e AIDS (16.516). A Organização Mundial de Saúde lançou em 2004 o programa "Aliança Mundial para Segurança do Paciente", no qual Canadá e Estados Unidos já fazem parte, e os países do Mercosul se articulam para também participar, estando o Brasil vinculado a partir de $2007^{(3)}$. Este programa inclui tópicos e campanhas de prevenção de infecções hospitalares e cirurgias seguras, buscando redução dos danos causados por procedimentos cirúrgicos.

No Brasil, o reconhecimento da segurança do paciente como necessidade e garantia de qualidade do atendimento, teve um impulso na década de 90, juntamente com o lançamento do Manual Brasileiro de Acreditação Hospitalar, e a criação da Organização Nacional de Acreditação, o que impulsionou trabalhos na área ${ }^{(4)}$. Em $1^{\circ}$ de abril de 2013, o Ministério da Saúde e Anvisa lançam o Programa Nacional de Segurança do Paciente, objetivando reduzir a incidência de EA nos serviços de saúde, que são altas, mas, em sua maioria, evitáveis ${ }^{(5)}$.

Um estudo analisando três hospitais no Rio de Janeiro identificou que oito em cada 100 pacientes internados sofreram EAs, sendo que $67 \%$ dessas ocorrências eram evitáveis. Mostrou ainda que o número de dias adicionais de permanência no hospital, em decorrência desses eventos, triplicou ocorrendo, com maior freqüência, em unidades de internamento, e que o procedimento cirúrgico foi a causa mais recorrente ${ }^{(6)}$.

Na Suécia, um trabalho realizado com 1,2 milhões de pacientes admitidos, mostrou taxa de EA de $8,6 \%$, sendo que a maioria destes ocorreu na população acima de 65 anos, poderiam ter sido evitados e resultaram em lesões permanentes ou contribuíram para o óbito(7).

Na Colômbia, a incidência de EA em 6.688 pacientes hospitalizados foi de $4,6 \%$, com predominância de idosos envolvidos, sendo o evento mais prevalente o erro técnico, com 57,5\% ocorrências. Nesse estudo, a Unidade de Terapia Intensiva (UTI), foi o local de menor ocorrência ${ }^{(8)}$. No Canadá, a estimativa de ocorrência de EA foi de 7,5\%, também com prevalência em idoso( ${ }^{(9)}$.

O trabalho em UTI é peculiar, pois as condições clínicas dos pacientes são oscilantes, exigindo atenção ainda maior da equipe multiprofissional. Um estudo avaliando 113 ocorrências iatrogênicas em sete UTIs gerais, mostrou que 57,5\% destes eventos ocorreram em pacientes que se encontravam com quadro clínico instável(10). O mesmo trabalho, em relação à idade, mostrou predomínio de EA em pacientes com idade a partir de 40 anos, correspondendo a $80,5 \%$ dos pacientes envolvidos em iatrogenias. Destes, $57,5 \%$ tinham mais que 60 anos. Outro estudo americano mostrou que a idade de risco para EA foi acima de 62.1 anos, com elevação do risco após 76.3 anos $^{(11)}$.

Resultado parecido foi encontrado em dois outros estudos: o primeiro, que objetivou caracterizar EAs em UTIs, Unidades Semi-intensivas e Unidades de Internação, observou que o grupo etário mais atingido foi, predominantemente, o de idade maior que 61 anos, correspondendo a $77,7 \%$ dos pacientes envolvidos na pesquisa ${ }^{(12)}$. O segundo, realizado com 30.000 prontuários de 51 hospitais de Nova lorque, identificou que pacientes com mais de 65 anos de idade, sofreram EA 2 vezes mais que os pacientes entre 16 a 44 anos. Além disso, esses eventos foram responsáveis por percentual significativo de incapacidades e mortes ${ }^{(13)}$.

Sabe-se que a população mundial está envelhecendo e que, nos países em desenvolvimento como o Brasil e países da America Latina, essa mudança na pirâmide demográfica tem ocorrido de forma acelerada, com pouco preparo de recursos humanos e materiais para atendê-la. Em 2025, haverá no mundo aproximadamente 1,2 bilhões de pessoas com mais de 60 anos e, até 2050, 2 bilhões, sendo que $80 \%$ nos países em desenvolvimento ${ }^{(14)}$.

O envelhecimento da população traz, como consequência, diminuição da mortalidade e aumento da morbidade, com o aparecimento de doenças crônicas não transmissíveis que, em uma situação de agudização, pode levar o idoso ao internamento na UTI.

Além disso, o envelhecimento está associado à diminuição das reservas fisiológicas e aumento do risco para desenvolvimento de doenças. Quando ocorre um processo agudo de doença ou estresse, geralmente o idoso tem menor capacidade fisiológica de superar as lesões, tornando-se mais vulnerável e frágil|(15).

Considerando a especificidade do cuidado em saúde da população idosa e os estudos publicados relacionados aos EAs, reitera-se a necessidade de discussão sobre a segurança dos pacientes idosos internados nas UTIs, e levanta-se uma questão: como está a produção científica, na América Latina, sobre a segurança do idoso internado na UTI? Quais são os EAs mais relatados? Visto que nesses países, esse tema é atual, com recentes produções na área, seguindo o perfil de enveIhecimento da população.

O trabalho em tela teve como objetivo o de identificar a produção cientifica na America Latina sobre a segurança 
do idoso na UTI e os tipos de eventos adversos trazidos pela literatura.

\section{MÉTODO}

Pesquisa bibliográfica, quantitativa, que se utilizou da revisão sistemática. Esta se caracteriza pela busca de informações na literatura sobre determinado tema, utilizando métodos sistematizados e explícitos de pesquisa, síntese e análise crítica ${ }^{(16)}$.

A coleta dos dados foi feita no banco de dados do LILACS e na Biblioteca da SCIELO, considerando-se os países da América Latina, a partir dos descritores indexados: segurança, iatrogenia, unidade de terapia intensiva e idoso, cruzados entre si. Como critérios de seleção, foram considerados todos os textos encontrados até 22 de dezembro de 2012, disponíveis de forma completa, em qualquer data de publicação. Foram excluídos estudos do tipo revisão.

A partir dessa busca, foram selecionados 259 artigos. Foi feita leitura dos resumos e aplicado como critério de inclusão: artigos que tratavam diretamente dos erros ou faltas cometidas pelos profissionais durante o cuidado prestado, considerando a segurança e as iatrogenias em idosos. Foram excluídos os artigos que tratavam sobre segurança e manutenção de equipamentos diversos, artigos de pediatria referentes às iatrogenias e textos que incluíam o tema terapia intensiva, porém não estavam relacionados ao objeto do estudo.

Os artigos selecionados foram lidos e fichados, buscando-se as variáveis independentes: ano, local do estudo, tipo de estudo, área de conhecimento e tipo de evento adverso citado.

\section{RESULTADO}

Foram selecionados vinte artigos para discussão, após aplicação do critério de inclusão. A maioria dos trabalhos analisados foram publicados a partir do ano 2000, no Brasil, no estado de São Paulo, realizados por profissionais médicos e enfermeiros, que utilizaram com mais freqüência a metodologia quantitativa. Quanto aos eventos adversos citados, houve prevalência daqueles relacionados à medicação, seguido de EAs variados, como perdas ou lesões decorrentes do uso de sondas e cateteres, retiradas não programadas de tubos, cateteres e drenos, exteriorização de sondas nasoenterais e nasogástricas, flebite, infiltração de acessos venosos, queda e falha na rede de vácuo. Depois destes, os eventos mais relatados se referiam à infecção nosocomial, úlcera por pressão, erro na técnica de procedimento diagnóstico ou terapêutico.

Quadro 1 - Perfil dos estudos encontrados nas bases de dados LILACS e SCIELO, após o cruzamento dos descritores idoso, segurança, iatrogenia e unidade de terapia intensiva até 2012.

\begin{tabular}{|c|c|c|c|c|c|c|}
\hline Titulo & Autor & Ano & Local de estudo & $\begin{array}{l}\text { Tipo de } \\
\text { estudo }\end{array}$ & $\begin{array}{c}\text { Área de } \\
\text { conhecimento }\end{array}$ & Tipo de EA citado \\
\hline $\begin{array}{l}\text { 1.Rev Latino-Am } \\
\text { Enfermagem }\end{array}$ & $\begin{array}{l}\text { Nascimento; } \\
\text { Toffoletto; Gonçalves; } \\
\text { Freitas; Padilha }\end{array}$ & 2008 & São Paulo & Quanti & Enfermagem & $\begin{array}{l}\text { Retirada não programada de } \\
\text { sonda nasogástrica e erro de } \\
\text { medicação. }\end{array}$ \\
\hline $\begin{array}{l}\text { 2.Rev Associação } \\
\text { Médica Brasileira }\end{array}$ & $\begin{array}{l}\text { Maurici; do } \\
\text { Valle; Zaclikevis; } \\
\text { Kleinubing Junior }\end{array}$ & 2007 & Santa Catarina & Quanti & Medicina & $\begin{array}{l}\text { Úlcera por pressão nas regiões } \\
\text { sacra, calcânea e isquiática. }\end{array}$ \\
\hline $\begin{array}{l}\text { 3.Arquivos de } \\
\text { gastroenterologia }\end{array}$ & $\begin{array}{l}\text { Borges; Pinheiro; } \\
\text { Pace; Chebli }\end{array}$ & 2008 & Minas Gerais & Quanti & Medicina & $\begin{array}{l}\text { Diarréia associada à exposição } \\
\text { a patógenos, procedimentos e } \\
\text { medicamento, principalmente } \\
\text { antibióticos em grande } \\
\text { quantidade. }\end{array}$ \\
\hline $\begin{array}{l}\text { 4.Rev. Bras. } \\
\text { Terapia Intensiva }\end{array}$ & $\begin{array}{l}\text { Santos; Omur; } \\
\text { Guimarães; Falcão; } \\
\text { Delascio; Saes. }\end{array}$ & 2006 & São Paulo & $\begin{array}{l}\text { Quali- } \\
\text { estudo } \\
\text { de caso }\end{array}$ & Medicina & $\begin{array}{l}\text { Perfuração de esôfago por sonda } \\
\text { nasoenteral }\end{array}$ \\
\hline $\begin{array}{l}\text { 5.Rev. Bras. } \\
\text { Terapia Intensiva }\end{array}$ & $\begin{array}{l}\text { Faria; Hoher; Borges, } \\
\text { Schifelbain; Dias; } \\
\text { Lisboa; Friedman. }\end{array}$ & 2007 & Rio Grande do Sul & Quanti & Medicina & $\begin{array}{l}\text { Pneumonia, infecção do trato } \\
\text { respiratório inferior, infecção do } \\
\text { trato urinário por stafilococos } \\
\text { aureus e pseudomonas } \\
\text { aeruginosa.. }\end{array}$ \\
\hline $\begin{array}{l}\text { 6. Revista de } \\
\text { Saúde Pública }\end{array}$ & $\begin{array}{l}\text { Carvalho-Filho; } \\
\text { Saporetti; Souza; } \\
\text { Arantes; Vaz; Hojaiji; } \\
\text { Alencar; Curiati }\end{array}$ & 1998 & São Paulo & Quanti & Medicina & $\begin{array}{l}\text { Procedimento diagnóstico e } \\
\text { terapêutico, úlceras por pressão, } \\
\text { quedas e fraturas. }\end{array}$ \\
\hline $\begin{array}{l}\text { 7.Arq. Brasileiro } \\
\text { de Cardiologia }\end{array}$ & $\begin{array}{l}\text { Copini, Alves, Costa, } \\
\text { Souza, Herrmann }\end{array}$ & 1998 & São Paulo & $\begin{array}{l}\text { Quali- } \\
\text { estudo } \\
\text { de caso }\end{array}$ & Medicina & $\begin{array}{l}\text { Dissecção da artéria circunflexa } \\
\text { durante cateterismo diagnóstico }\end{array}$ \\
\hline $\begin{array}{l}\text { 8.Rev. Eletrônica } \\
\text { de Enferm. }\end{array}$ & Freire,Farias, Ramos & 2006 & Rio Grande do Norte & Quanti & Enfermagem & Pneumonia associada a VM \\
\hline $\begin{array}{l}\text { 9. Revista Latino- } \\
\text { Americana de } \\
\text { Enfermagem }\end{array}$ & Fernandes; Caliri & 2008 & São Paulo & Quanti & Enfermagem & $\begin{array}{l}\text { Úlcera por pressão em calcâneo } \\
\text { sacra e escápula }\end{array}$ \\
\hline
\end{tabular}




\begin{tabular}{|c|c|c|c|c|c|c|}
\hline $\begin{array}{l}\text { 10. Ciencia Cuid } \\
\text { Saude }\end{array}$ & $\begin{array}{l}\text { Myata, Molena- } \\
\text { Fernandes, Teixeira, } \\
\text { Silva, Tasca, Cuman }\end{array}$ & 2007 & Paraná & Quanti & $\begin{array}{c}\text { Medicina, } \\
\text { Farmácia, } \\
\text { Educação Física }\end{array}$ & Uso de medicação \\
\hline $\begin{array}{l}\text { 11. Revista } \\
\text { da Escola de } \\
\text { Enfermagem } \\
\text { da USP }\end{array}$ & Santos e Ceolin & 2007 & São Paulo & Quanti & Enfermagem & $\begin{array}{l}\text { Perda, infiltração, flebite ou } \\
\text { obstrução de acesso venoso } \\
\text { periférico e da SNE, hematoma } \\
\text { na parede abdominal, ulcera } \\
\text { por pressão, queda, erro com } \\
\text { medicação, sinais flogísticos em } \\
\text { cateter central. }\end{array}$ \\
\hline $\begin{array}{l}\text { 12. Revista da } \\
\text { Associação } \\
\text { Médica Brasileira }\end{array}$ & $\begin{array}{l}\text { Júnior, Haiashi, } \\
\text { Vidonho Júnior, } \\
\text { Abensur, Quintaes, } \\
\text { Araújo, Noronha, } \\
\text { Santos, Machado }\end{array}$ & 2000 & São Paulo & Quanti & Medicina & Nefrotixicidade \\
\hline $\begin{array}{l}\text { 13. Revista } \\
\text { Paulista de } \\
\text { Enfermagem }\end{array}$ & Padilha & 2006 & São Paulo & $\begin{array}{l}\text { Quanti- } \\
\text { quali }\end{array}$ & Enfermagem & $\begin{array}{l}\text { Perda do tubo traqueal, cateteres, } \\
\text { sondas e drenos. Erro com } \\
\text { medicação, troca da prescrição, } \\
\text { ausência de registro de dados do } \\
\text { balanço hídrico, e infusão de } \\
\text { soluções incompatíveis }\end{array}$ \\
\hline $\begin{array}{l}\text { 14. Universidade } \\
\text { do Estado do } \\
\text { Rio de Janeiro - } \\
\text { Dissertação de } \\
\text { Mestrado }\end{array}$ & Melo & 2007 & Rio de Janeiro & Quanti & Enfermagem & Erro com medicação \\
\hline $\begin{array}{l}\text { 15. Escola Anna } \\
\text { Nery }\end{array}$ & $\begin{array}{l}\text { Sousa; Alves; Santo.; } \\
\text { Santana }\end{array}$ & 2010 & Rio de Janeiro & Quanti & Enfermagem & $\begin{array}{l}\text { Risco de infecção, hipotermia e } \\
\text { hipertermia, integridade da pele } \\
\text { prejudicada, constipação, volume } \\
\text { de líquido deficiente, diarreia e } \\
\text { nutrição desequilibrada: menos } \\
\text { do que as necessidades diárias }\end{array}$ \\
\hline $\begin{array}{l}\text { 16. Revista } \\
\text { Brasileira de } \\
\text { Geriatria e } \\
\text { Gerontologia }\end{array}$ & $\begin{array}{l}\text { Lucchetti; Lamas.; } \\
\text { Gorzoni }\end{array}$ & 2010 & Rio de Janeiro & Quanti & Medicina & $\begin{array}{l}\text { Polifarmácia em idosos e } \\
\text { associação com quedas, } \\
\text { prescrição de medicamentos } \\
\text { inapropriados, desnutrição e } \\
\text { qualidade do sono. }\end{array}$ \\
\hline $\begin{array}{l}\text { 17. Faculdade } \\
\text { de Medicina da } \\
\text { Universidade de } \\
\text { São Paulo, Tese } \\
\text { de Doutorado }\end{array}$ & Szleijf & 2010 & São Paulo & Quanti & Medicina & $\begin{array}{l}\text { Delirium, infecção, medicamentos } \\
\text { inapropriados erros de } \\
\text { procedimentos ou diagnósticos, } \\
\text { complicações cirúrgicas. }\end{array}$ \\
\hline $\begin{array}{l}\text { 18. Revista } \\
\text { Brasileira Terapia } \\
\text { Intensiva }\end{array}$ & Silva; Paço.; Reis & 2011 & Rio de Janeiro & $\begin{array}{l}\text { Quali - } \\
\text { estudo } \\
\text { de caso }\end{array}$ & Medicina & $\begin{array}{l}\text { Infecção e sepse associada a } \\
\text { cuidados de saúde com quebra de } \\
\text { barreira de mucosa gastrointestinal } \\
\text { e utilização prévia do probiótico } \\
\text { Saccharomyces boulardii e } \\
\text { obstipante loperamida. }\end{array}$ \\
\hline $\begin{array}{l}\text { 19. Revista } \\
\text { Médica do Chile }\end{array}$ & $\begin{array}{l}\text { Plaza; Álamo; Torres; } \\
\text { Fuentes,; López }\end{array}$ & 2010 & Chile & Quanti & Medicina & $\begin{array}{l}\text { Erros com medicação: interações } \\
\text { medicamentosas com uso de } \\
\text { fármacos contra-indicados } \\
\text { e potencialmente perigosos } \\
\text { ocorrendo bradicardia, } \\
\text { hipotensão, retenção urinária } \\
\text { e síndrome de desconforto } \\
\text { respiratório agudo }\end{array}$ \\
\hline $\begin{array}{l}\text { 20. Revista } \\
\text { da Escola de } \\
\text { Enfermagem } \\
\text { da USP }\end{array}$ & $\begin{array}{l}\text { Gonçalves, Andolhe, } \\
\text { Oliveira, Barbosa, } \\
\text { Faro, Gallotti e } \\
\text { Padilha }\end{array}$ & 2012 & São Paulo & $\begin{array}{l}\text { Quanti- } \\
\text { quali }\end{array}$ & Enfermagem & $\begin{array}{l}\text { Falha de registro em prontuário, } \\
\text { erro no preparo e administração } \\
\text { de medicamentos, falha } \\
\text { relacionada à coleta e/ou } \\
\text { encaminhamento dos exames } \\
\text { ao laboratório de análises } \\
\text { clínicas, hipoglicemia e falha na } \\
\text { administração de dieta }\end{array}$ \\
\hline
\end{tabular}

*Médico, Educador Físico e Farmacêutico 


\section{DISCUSSÃO}

Nos últimos anos têm crescido o interesse das instituições de saúde em melhorar a segurança na prestação do cuidado, principalmente pelas discussões sobre a gestão da qualidade nos serviços de saúde ${ }^{(4)}$.

Nesse trabalho, os estudos foram encontrados após o ano de 1998. Os anos 90 marcaram abordagens sensacionalistas sobre erros na saúde, com aumento do numero de processos judiciais e do valor financeiro das indenizações ${ }^{(17)}$. Além disso, os estudos sobre iatrogenias tiveram inicio em 1980 por pesquisadores americanos em Harvard, só tendo impulso no Brasil e países da América Latina a partir de $90^{(10)}$.

No que se refere à origem da produção, apesar da pouca produção cientifica sobre o tema, gerada nos países da América Latina com exceção do Brasil, nos bancos de dados consultados, existe uma mobilização nestes no que se refere à qualidade dos cuidados em saúde. Em 1992, houve a publicação do Manual de Credenciamento para Hospitais da América Latina e do Caribe ${ }^{(17)}$. Além disso, em 2001, o México iniciou uma "Cruzada nacional pela qualidade", com o objetivo de elevar a qualidade dos serviços a níveis aceitáveis em todo o país: o Peru introduziu um sistema nacional de credenciamento de centros de saúde, com uma nova proposta implementada em 2007; a Argentina possui varias entidades dedicadas à melhoria da qualidade; a Costa Rica liderou iniciativas ligadas à segurança do paciente; a Colômbia dispõe de um sistema de notificação de EA e o Chile conta com sólida trajetória de iniciativas para o controle de infecções ${ }^{(19)}$.

Com relação ao Brasil, sua produção científica vem se destacando, não só em relação ao tema segurança, mas, principalmente, em relação à temática do envelhecimento, com trabalhos de colaboração e publicação nacional e internacional. A produção científica brasileira esta atrelada ao crescimento da pós-graduação, e este Pais encontra-se em $13^{\circ}$ lugar no ranking mundial de artigos publicados em revistas especializadas, sendo o país que mais cresceu na lista das 20 nações com mais artigos publicados em periódicos científicos indexados pelo Information Sciences Institute-ISI ${ }^{(20-21)}$.

No Brasil, o sudeste tem se destacado com um grande numero de produções não só nessa área. Essa região possui o maior número de idosos no Brasil, com destaque para o Estado de São Paulo. A região Nordeste, que aparece no estudo somente com uma publicação, é a segunda região em número de idosos no País, e a primeira em relação ao numero de idosos com mais de 100 anos $^{(22)}$.

O fato de a região Sudeste concentrar maior percentual de artigos pode denotar o interesse em pesquisas sobre o processo de envelhecimento e as questões relativas a este grupo específico. Além disso, essa região possui grande numero de grupos de pesquisa e pesquisadores cadastrados, com 59,7\% dos grupos de pesquisa sobre envelhecimento. A região Sul possui $21,5 \%$ e o Nordeste abriga apenas 13,9\% dos grupos de pesquisa sobre idoso. No Sudeste também se concentram maior numero de grupos de pesquisa em segurança do paciente cadastrados no CNPq, principalmente em São Paulo ${ }^{(23)}$.

Embora não tenha sido objetivo do estudo levantar os autores dos trabalhos publicados, é importante apontar que, muitos dos estudiosos sobre a temática no Brasil, são pesquisadores locados também na região sudeste, influenciando a formação desses grupos de pesquisa.

No trabalho em tela, houve predominância de trabalhos publicados por profissionais enfermeiros e médicos. Uma pesquisa nacional sobre a produção científica em relação ao processo de envelhecimento no Brasil constatou que a área da saúde coletiva, enfermagem e medicina são as que possuem maior número de grupos com linhas de pesquisa específicas sobre idoso ${ }^{(23)}$.

Esse tema também está muito relacionado com o cotidiano da prática de enfermagem. Um grande número de EAs ocorre com o envolvimento direto da equipe de enfermagem, como verificado em outros estudos ${ }^{(7-9)}$.

Nesse sentido, entre os temas discutidos pelos autores, os mais abordados pela enfermagem foram: eventos adversos à medicação (5), eventos adversos variados (4), infecção nosocomial (3), ulceras por pressão (2) e erro na técnica de procedimento (2).

Nas instituições hospitalares, o envolvimento das enfermeiras com falhas na prestação de cuidados aos pacientes pode ser evidenciado em situações como: erros de medicação, queda do paciente, extubação, queimaduras durante procedimentos, hemorragias por desconexão de drenos e cateteres dentre outros $^{(12)}$. Em relação às falhas médicas, pode-se citar erros de diagnostico, lesões causadas por procedimentos terapêuticos e falhas na prescrição ${ }^{(17)}$.

As questões éticas e jurídicas também podem explicar o interesse crescente das áreas da medicina e enfermagem em segurança do paciente. Com a criação do Código de Defesa do Consumidor, os pacientes tornaram-se consumidores conscientes dos seus direitos. E os processos judiciais por erro médico têm sido mais frequentes nos tribunais brasileiros ${ }^{(24)}$.

No que se refere à publicação da equipe multiprofissional, só foi encontrado um trabalho, que envolveu o médico, o educador físico e o farmacêutico. É importante incentivar a produção multiprofissional envolvendo esse tema, pois, em muitas ocasiões, a ocorrência de EA é multifatorial, envolvendo mais de um membro da equipe, principalmente se estiver ligado ao uso do medicamento ${ }^{(1,10,17)}$.

Em relação ao método, cabe ressaltar que esse estudo objetivou apenas citar o método utilizado, sem avaliá-lo. Dessa forma, observou-se na Enfermagem um número maior de estudos com metodologia qualitativa, o que, certamente, se justifica pela subjetividade do cuidado de enfermagem e pelos temas a ela relacionados. Os três estudos quanti-qualitativos também foram publicados pela Enfermagem, mostrando que, mesmo nos estudos quantitativos realizados nessa área, existe uma preocupação com a abordagem qualitativa. Nos oito estudos realizados pela medicina, houve predominância do método quantitativo, utilizado em seis deles.

A investigação quantitativa gera dados, indicadores e tendências observáveis, ao passo que a investigação qualitativa, trabalha com crenças, representações, valores, opiniões. A combinação das duas abordagens pode gerar resultados ainda mais completos. A segurança do idoso hospitalizado em UTI é um tema que pode revelar inúmeras falhas no processo de 
prestação do cuidado. Portanto, uma abordagem metodológica quantitativa ajuda a responder questões sobre os tipos de EAs, sua frequência e população envolvida. Contudo, a abordagem qualitativa pode ser interessante para explicar os fatores determinantes e o impacto destes na vida das pessoas e serviços envolvidos.

No que se refere aos tipos de EAs encontrados, os erros com medicação tiveram destaque. Esses dados corroboram resultados apresentados em outros estudos ${ }^{(1,7-9,11)}$. Observa-se assim que, infelizmente, esses erros são comuns, impõem custos importantes ao sistema e são clinicamente relevantes.

Os erros com medicação podem se relacionar ao exercício profissional; ao procedimento em si; aos problemas de comunicação, incluindo prescrição, rótulos, embalagens, nomes, preparação, dispensação, distribuição, administração e formação (poucos intensivistas têm preparo para trabalhar com idosos) e ao monitoramento e uso de outros medicamentos, fato comum nessa população ${ }^{(25-26)}$. No idoso, as alterações fisiológicas do envelhecimento como o declínio das funções hepática e renal aumentam os riscos envolvendo medicamentos.

Dados do livro "Errar é Humano"(1), demonstrou que os erros devido às medicações causam aproximadamente 7.391 óbitos anuais de americanos nos hospitais. Um estudo americano, que observou 5.744 administrações de medicamentos em cinco UTIs, revelou que o erro mais comum envolvia velocidade errada de infusão ${ }^{(26)}$. Esse tipo de erro pode ocorrer em todas as fases que compõem o processo, como armazenamento, distribuição, prescrição e administração. Por ser uma problemática que envolve não só a Enfermagem, mas, também, farmacêuticos, médicos e auxiliares administrativos, o EA com medicação torna-se uma questão multiprofissional e suas causas são multifatoriais. Dessa forma, a abordagem deve ser centrada em todas as fases mencionadas, sendo importante também considerar as condições nas quais esses profissionais trabalham e sua formação ${ }^{(10,25,27)}$.

Um trabalho que procurou identificar situações facilitadoras dos erros e analisar as ocorrências dos mesmos nas diversas etapas que envolvem a administração de medicamentos encontrou que: o ambiente de preparo ruidoso, a ausência no esclarecimento ao paciente sobre o procedimento e a administração de medicamentos por ordem verbal, foram as situações facilitadoras de erros mais frequentes, com 68\%, 64\% e $40 \%$ respectivamente. Além disso, a ausência de protocolos de administração de drogas endovenosas correspondeu a $100 \%$ dos casos de erros, a ausência de registro da administração foi responsável por $48 \%$ e o atraso na administração de medicamentos obteve $34 \%$ dos $\operatorname{erros}^{(28)}$.

Nesse sentido, a OMS possui um programa internacional de medicamentos do qual o Brasil faz parte desde 2001 e, desde então, a Agência Nacional de Vigilância Sanitária (ANVISA) vem desenvolvendo diversas iniciativas de fármaco-vigilância, como a criação da rede de hospitais sentinelas e, mais recentemente, em 2003, do Sistema de Informação de Notificação de Evento Adverso e Queixas Técnicas Relacionados a Produtos de Saúde, o SINEPS ${ }^{(29)}$.

Em relação aos EAs variados, que incluíram perda ou lesões relacionadas ao uso de sondas e cateteres, retiradas não programadas de tubos, cateteres e drenos, exteriorização de sondas nasoenterais e nasogástricas, flebite, infiltração de acessos venosos, queda e falha na rede de vácuo, cabe ressaltar que alguns fatores estruturais da UTI, a gravidade e a idade destes pacientes, bem como a quantidade de dispositivos que utilizam, interferem ${ }^{(10,12-13)}$. Um estudo objetivando identificar os fatores estruturais da UTI e as condições do paciente relacionado às ocorrências iatrogênicas, e verificar a associação entre a gravidade destas e os fatores relacionados, identificou que, quanto à natureza, dos 113 eventos, 46\% ocorreu devido a acidentes com cateteres, $40 \%$ com tubos endotraqueais e $7 \%$ com sondas e drenos ${ }^{(10)}$.

O controle de infecções é uma realidade constante no ambiente hospitalar, principalmente pelo grande número de antibióticos utilizados e o consequente aumento de infecções causadas por bactérias multirresistentes. Portanto, há uma preocupação mundial no controle destas, nos danos gerados para o paciente que as adquire e também em relação aos custos decorridos destas infecções. Nesse estudo, dois publicações abordaram esse tema.

As infecções hospitalares aumentam os riscos de morbimortalidade, o tempo de estadia e os custos durante a internação. No idoso, estas assumem uma importância maior em decorrência da gravidade destes quando internados na $U \mathrm{TI}^{(30)}$. Um estudo observou que a infecção hospitalar foi o evento mais prevalente, ocorrendo em 21,8\% da amostra, a frente de acidentes e leões, observado em 11,6\%(31). Importante destacar que as infecções mais comuns nessas unidades, estão relacionadas ao trato respiratório e urinário, acometidas pelo stafilococos aureus e pseudomonas aeruginosa ${ }^{(32)}$.

A ocorrência de ulceras por pressão como iatrogenia pode, muitas vezes, ser evitada através do cuidado direto da enfermagem. No presente estudo a discussão sobre este EA é importante, não só pela peculiaridade com a profissão, mas por se tratar do acometimento de úlceras por pressão em idosos, tornando sua incidência ainda mais elevada.

A epidemiologia das úlceras por pressão é elevada, com incidência de 2,7 a 29\% entre os pacientes hospitalizados e $33 \%$ nos pacientes internados em UTIs, destacando-se como regiões mais acometidas: calcâneo, sacra, escápula e ísquio ${ }^{(33)}$. Estes dados são globais e podem ser mais elevados dependendo do local e da população estudada ${ }^{(34)}$. Contudo, em outros estudos, não tiveram o mesmo destaque ${ }^{(7-9,11)}$.

Os erros relacionados a falhas técnicas nos procedimentos estiveram presentes em 12,5\% dos artigos selecionados. Esse evento adverso foi também citado em outros estudos, onde esteve significativamente presente ${ }^{(7-9)}$.

Cabe ressaltar, sobre os erros acima destacados, que o Programa Nacional de Segurança do Paciente traz seis protocolos: higienização das mãos, cirurgia segura, prevenção de úlcera por pressão, identificação do paciente, prevenção de quedas e prescrição, uso e administração de medicamentos, que devem ser trabalhados nas instituições de saúde para reduzir danos aos pacientes hospitalizados ${ }^{(5)}$.

Chamou atenção que somente um trabalho tenha relatado EA relacionado a alterações comportamentais no paciente, trazendo a ocorrência de delirium, vez que eventos adversos 
ligados a alterações comportamentais, foram citados em estudos internacionais ${ }^{(6,8)}$, mostrando-se relevantes. Considerando que, na UTI, a população idosa esta mais suscetível a delirium, depressão, perda da identidade, desorientação, entre outras alterações comportamentais, e que estas podem acarretar diversas outras situações como não aceitação do tratamento e/ou cuidados e quedas, cabe atentar também para esse tipo de EA.

\section{CONSIDERAÇÕES FINAIS}

O estudo buscou respostas para as seguintes questões: como está a produção científica, na América Latina, sobre a segurança do idoso internado na UTI e quais são os EAs mais relatados nessa produção? Observou-se que a produção sobre o tema tem crescido na América Latina, principalmente a partir do ano 2000, no Brasil, na região sudeste, contribuindo para isso o aumento da população idosa e dos grupos de pesquisa sobre envelhecimento e segurança do paciente e a introdução de discussões sobre o tema no país a partir dos anos 1990.

Em relação aos profissionais, houve predominância de trabalhos publicados pela Enfermagem e Medicina, sendo que a Enfermagem focou os EAs relacionados a erros com medicação, infecção nosocomial, úlceras por pressão e erro na técnica de procedimento. A produção da Medicina foi direcionada a EAs durante procedimentos médicos diagnósticos e terapêuticos.

Dos trabalhos analisados, somente um trouxe o delirium como EA na UTI, indo ao encontro de estudos internacionais que apontam as alterações comportamentais como importante EA a ser reconhecido.

Recomenda-se a realização de pesquisas em campo, envolvendo a equipe multiprofissional, atentando também para as iatrogenias que podem gerar alterações psicológicas e comportamentais no idoso hospitalizado.

\section{REFERÊNCIAS}

1. Mendes W, Travassos C, Martins M, Noronha JC. Revisão dos estudos de avaliação da ocorrência de eventos adversos em hospitais. Rev Bras Epidemiol 2005;8(4):393-406.

2. Kohn KT, Corrigan JM, Donaldson MS, editores. To err is human: Building a safer health system. Washington: National Academy Press, 1999.

3. World Health Organization. World alliance for patient safety: forward programme 2006-2007 [monografia na internet]. Geneva; 2006 [acesso em 29 dez 2007]. Disponível em: http://www.who.int/patientsafety/en.

4. Toffolleto, MC, Silva SC, Padilha KG. Segurança do paciente e prevenção de eventos adversos em UTI. In: Padilha KG, Vattimo, MFF, Silva, SC. Enfermagem em UTI: cuidando do paciente crítico. Baueri: Mamole; 2010. p. 1171-1189.

5. Brasil. Ministério da Saúde. ANVISA. Portaria n. ${ }^{\circ}$ 529, de 1 de abril de 2013. Institui o Programa Nacional de Segurança do Paciente (PNSP) [portaria na internet]. Diário Oficial da União 02 abr 2013 [acesso em 27 fev 2011];Seção1. Disponível em: bvsms.saude.gov.br/bvs/saudelegis/ gm/2013/prt0529_01_04_2013.html.

6. Brasil. Ministério da Saúde. Fiocruz. Revista ICICT [periódico na internet] 2009 [acesso em 27 fev 2011];4(4). Disponivel em: http://www.fiocruz.br/cict/media/revista_online_2009.pdf.

7. Soop M, Fryksmark U, Koster M, Haglund B. The incidence of adverse events in Swedish hospitals: a retrospective medical record review study. Int J Qual Health Care 2009;21(4):285-291.

8. Gaitán-Duarte H, Eslava-Schmalbach J, Rodríguez-Malagon N, Forero-Supelano V, Santofimio-Sirra D, Altahona $\mathrm{H}$. Incidence and preventability of adverse events in patients hospitalised in three Colombian hospitals during 2006. Rev Salud Pública 2009;10(2):215-226.
9. Baker GR, Norton PG, Flintoft V, Blais R, Brown A, Cox J, et al. The Canadian Adverse Events Study: the incidence of adverse events among hospital patients in Canada. CMAJ 2004;170(11):1678-1686.

10. Padilha KG. Ocorrências iatrogênicas em Unidade de Terapia Intensiva (UTI): análise dos fatores relacionados. Rev Paul Enferm 2006;25(1):18-23.

11. Chaboyer W, Thalib L, Foster M, Ball C, Richards B. Predictors of adverse events in patients after discharge from the intensive care unit. Am J Crit Care 2008;17(3):255-263.

12. Nascimento CCPN, Toffoletto MC, Gonçalves LA, Freitas WG, Padilha, KG. Indicadores de resultados da assistência: análise dos eventos adversos durante a internação hospitalar. Rev Latino-Am Enfermagem [periódico na internet]. 2008 Ago [acesso em 14 jan 2011 ];16(4):746-751. Disponível em: http://www.scielo.br/scielo.php?pid=s0104$-11692008000400015 \&$ script $=$ sci_abstract\&tlng $=$ pt.

13. Brennan TA, Leape LL, Laird NM, Hebert L, Localio AR, Lawthers AG, et al .Incidence of adverse events and negligence in hospitalized patients: results of the Harvard medical practice study I. N Engl J Med 1991;324(6):370-6.

14. Organização Mundial de Saúde [homepage na internet]. Plan de Acción Internacional de Madrid sobre el envejecimiento. [acesso em 27 fev 2011 ]. Disponível em: http://social.un.org/ageing-working-group/documents/ mipaa-sp.pdf

15. Knobel E. Condutas no Paciente Grave. 3. ed. São Paulo: Atheneu; 2006.

16. Sampaio RF, Mancini MC. Estudos de revisão sistemática: um guia para síntese criteriosa da evidência científica. Rev Bras Fisioter 2007;11(1):83-89.

17. Rosa MB, Perini E, Anacleto TA, Neiva HM, Bogutchi T. Erros na prescrição hospitalar de medicamentos potencialmente perigosos. Rev Saúde Pública [periódico na internet]. 
2009 jun [acesso em 29 dez 2010];43(3):490-498. Disponível em: http://www.scielo.br/scielo.php?pid=S0034$-89102009000300013 \&$ script $=$ sci_abstract\&tlng $=$ pt.

18. Organização Mundial da Saúde [homepage na internet]. $27^{\text {a }}$ Conferência Sanitária Pan-Americana.Política e estratégia regional para garantia da qualidade da atenção de saúde, inclusive a segurança do paciente. [acesso em 27 fev 2011]. Disponível em http://www.paho.org/portuguese/gov/csp/csp27-16-p.pdf.

19. Pan american Health Organization [homepage na internet]. Hospital accreditation for Latin America and the Caribbean. [acesso em 27 fev 2011]. Disponível em: http://bases.bireme.br/cgi-bin/wxislind.exe/iah/online/?lsisScript = iah/iah.xi $\mathrm{s} \& \mathrm{src}=$ google $\&$ base $=$ LILACS\&lang $=p \&$ nextAction $=$ Ink $\&$ exprSearch $=386282 \&$ indexSearch $=$ ID

20. Marziale MHP. Produção científica da enfermagem brasileira: a busca pelo impacto internacional. Rev Latino-Am Enferm [periódico na internet]. 2005 jun [acesso em 14 jan 2011];13(3):285-6. Disponível em: http://www.scielo. br/pdf/rlae/v13n3/v13n3a01.pdf.

21. Goes A. Produção científica cresce $56 \%$ no Brasil. Folha de São Paulo[ periódico na internet] 2009 [acesso em 06 maio 2009]. Disponivel em: http://www1.folha.uol.com. br/folha/ciencia/ult306u561181.shtml

22. Instituto Brasileiro de Geografia e Estatística [homepage na internet]. Censo 2009 [acesso em 01 dez 2010]. Disponível em http://www.ibge.gov.br/

23. Prado SD, Sayd JD. A pesquisa sobre envelhecimento humano no Brasil: grupos e linhas de pesquisa. Ciênc Saúde Coletiva 2004:9(1):57-68.

24. Carvalho BR, Ricco RC, Santos R, Campos MAF, Mendes ES, Mello ALS, et al. Erro médico: implicações éticas, jurídicas e perante o código de defesa do consumidor. Rev Ciênc Méd 2006;15(6):539-546.

25. Rosa MB, Perini E. Erros de medicação: quem foi?. Rev Assoc Med Bras [periódico na internet]. 2003 jun [acesso em 14 jan 2011];49(3):335-341. Disponível em: http://bases. bireme.br/cgi-bin/wxislind.exe/iah/online/?lsisScript = iah/ iah. xis\&nextAction $=$ Ink\&base $=$ LILACS\&exprSearch $=34$ 9577\&indexSearch $=$ ID\&lang $=p$

26. Calabrese AD, Erstad BL, Brandl K, Barletta JF, Kane SL, Sherman DS. Medication administration errors in adult patients in the ICU. Intensive Care Med 2001;27(10):1592-1598.

27. Franco JN, Ribeiro G, D'Innocenzo M, Barros BPA. Percepção da equipe de enfermagem sobre fatores causais de erros na administração de medicamentos. Rev Bras Enferm. [periódico na Internet]. 2010 dez [acesso em 24 fev 2011];63(6):927-932. Disponível em http://www.scielo.br/scielo.php?script=sci_arttext\&pid $=$ S0034-71672010000600009.

28. Melo ABR, Silva LD. Segurança na terapia medicamentosa: uma revisão bibliográfica. Esc Anna Nery Rev Enferm 2008;12(1):166-72.

29. Agencia Nacional de Vigilância Sanitária [homepage na internet]. Sistema de Informação de Notificação de Eventos Adversos e Queixas Técnicas relacionados a Produtos de Saúde [acesso em 27 fev 2011]. Disponível em http://www.anvisa.gov.br/servicosaude/hsentinela/sineps/ index.htm

30. Stein FC, Barros RK, Feitosa FS, Toledo DO, Silva Junior JM, Ísola AM, et al . Fatores prognósticos em pacientes idosos admitidos em unidade de terapia intensiva. Rev Bras Ter Intensiva [periódico na internet]. 2009 ago [acesso em 18 jan 2011];21(3):255-261. Disponível em: http://www.scielo.br/pdf/rbti/v21n3/a04v21n3.pdf

31. Chaboyer W, Thalib L, Foster M, Ball C, Richards B. Predictors of adverse events in patients after discharge from the intensive care unit. Am J Crit Care 2008;17(3):255-63; quiz 264.

32. Lisboa T, Faria M, Hoher JA, Borges LAA, Gómez J, Schifelbain L, et al. Prevalência de infecção nosocomial em unidades de terapia intensiva do Rio Grande do Sul. Rev Bras Ter Intensiva [periódico na internet]. 2007 ago [acesso em 18 jan 2011];19(4):414-420. Disponível em: http://www.scielo.br/scielo.php?script =sci arttext\&pid $=$ S0103-507X2007000400002

33. Fernandes LM, Caliri, MHL. Uso da escala de Braden e de Glasgow para identificação do risco para úlceras de pressão em pacientes internados em centro de terapia intensiva. Rev Latino-Am Enferm [periódico na internet]. 2008 ago [acesso em 18 jan 2011];16(6):33-38. Disponível em: http://www.scielo.br/pdf/rlae/v16n6/pt_06

34. Rocha JA, Miranda MJ, Andrade MJ. Abordagem terapêutica das úlceras de pressão - Intervenções baseadas na evidência. Acta Med Port 2006;19:29-38. 